\title{
Die ethische Aufsicht über die Datenwissenschaft im Gesundheitswesen
}

\author{
Stuart McLennan
}

\section{$1 \quad$ Einführung}

Die evidenzbasierte Medizin entstand im zwanzigsten Jahrhundert und betont die Bedeutung der Integration von klinischem Fachwissen mit der besten verfügbaren Evidenz (Sackett 1997). Zwar hat die evidenzbasierte Medizin zu großen Fortschritten geführt, doch gibt es nach wie vor erhebliche ungerechtfertigte Unterschiede zwischen den Behandlungen, die Kliniker und Gesundheitssysteme routinemäßig in der Praxis durchführen, sowie Defizite bei allen Schlüsselaspekten einer qualitativ hochwertigen Gesundheitsversorgung (Institute of Medicine 2001; Institute of Medicine 2013). Der unzureichende Schutz der Patienten vor ungerechtfertigten Schäden und Belastungen durch die klinische Versorgung wurde als ein ,zutiefst ernstes moralisches Problem” identifiziert (Faden et al. 2013). Teilweise als Folge dieser Situation nutzen Gesundheitssysteme auf der ganzen Welt zunehmend die große Menge routinemäßig erhobener digitaler Gesundheitsdaten, um die kontinuierliche Verbesserung der Qualität und Sicherheit der Gesundheitsversorgung zu gewährleisten und Fragen zur Wirksamkeit und Effektivität der Behandlung zu beantworten (Hemkens et al. 2016). Obwohl diese Arbeit dringend notwendig ist, gibt es Herausforderungen in Bezug auf die ethische Aufsicht. Dazu gehört die Entscheidung, welche Aktivitäten einer ethischen Überprüfung bedürfen (Morain und Kass 2016; Fiscella et al. 2015; Finkelstein et al. 2015; Kass und Pronovost 2011), und wann die Teilnehmer informiert und um ihre informierte Zustimmung gebeten werden sollten (Fiscella et al. 2015; Finkelstein et al. 2015; Andersonet al. 2015; Pletcher et al. 2014; Mostert et al. 2016). In diesem Artikel werden zuerst die Defizite

S. McLennan $(\bowtie)$

Institut für Geschichte und Ethik der Medizin, Technische Universität München,

München, Deutschland

E-Mail: stuart.mclennan@tum.de

G. Richter et al. (Hrsg.), Datenreiche Medizin und das Problem der Einwilligung,

https://doi.org/10.1007/978-3-662-62987-1_4 
bei der Qualität und Sicherheit der Gesundheitsfürsorge und die enormen menschlichen und finanziellen Konsequenzen untersucht, die sich daraus ergeben können. Dann wird er die Entstehung des Konzepts "Learning Health Care" als Antwort auf diese Defizite und die Rolle der Datenwissenschaft diskutieren. Als Beispiel für den Einsatz der Datenwissenschaft im Gesundheitswesen zur Verbesserung von Qualität und Sicherheit wird die Datenbank Medical Information Mart for Intensive Care (MIMIC) vorgestellt. Schließlich wird er sich mit Fragen der ethischen Aufsicht über die Datenwissenschaft in der Gesundheitsversorgung befassen, einschließlich der Unterscheidung Forschung vs. Nicht-Forschung sowie Patienteneinwilligung und Forschungsausnahmen. Insbesondere wird er die Herausforderungen im Zusammenhang mit der Datenwissenschaft im Gesundheitswesen und der Datenschutz-Grundverordnung (DSGVO) sowie die aktuelle COVID-19-Pandemie erörtern.

\section{Qualität und Sicherheit der Gesundheitsversorgung}

Die Frage der Patientensicherheit und der Qualitätsverbesserung in der Gesundheitsversorgung ist $\mathrm{zu}$ einem zentralen Anliegen der Gesundheitssysteme in aller Welt geworden, insbesondere seit das Institute of Medicine im Jahr 2000 in seinem ersten Bericht “To Err Is Human” alarmierende Statistiken über die Häufigkeit, die Schäden und die Kosten medizinischer Fehler veröffentlicht hat (Institute of Medicine 2000). Der Bericht schätzte beispielsweise, dass jedes Jahr zwischen 44.000 und 98.000 Personen an medizinischen Fehlern in Krankenhäusern der Vereinigten Staaten sterben. Damit wären medizinische Fehler mindestens die achthäufigste Todesursache in den Vereinigten Staaten. Die New York Times verglich dies mit dem Äquivalent von drei Jumbo-Jets, die alle zwei Tage abstürzen. Wie der Reporter bemerkte: „Wenn die Fluggesellschaften jährlich so viele Menschen töten würden, würde die öffentliche Empörung sie über Nacht schließen“ (zitiert in Banja 2005, S. 2). Zwar gab es anfänglich Widerstand gegen die Statistiken des Berichts über die Zahl der Todesfälle im Zusammenhang mit medizinischen Fehlern, ,diese Infragestellungen wurden jedoch durch das Überwiegen der Beweise dafür, dass die Rate schädlicher medizinischer Fehler - mit ihren enormen menschlichen und finanziellen Folgen in Form von Tod, Behinderung, Einkommensverlust, Produktionsausfall im Haushalt und Gesundheitskosten - inakzeptabel ist, zum Schweigen gebracht“" (Sharpe 2004, S. 2; meine Übersetzung S.M.).

Tatsächlich legen neuere Untersuchungen nahe, dass der Bericht des Instituts für Medizin das Ausmaß des Problems deutlich unterschätzt hat. Im Jahr 2016 berechneten Makary und Daniel eine durchschnittliche Sterblichkeitsrate aufgrund von medizinischen Fehlern in US-Krankenhäusern von 251.454 pro Jahr, was darauf hindeuten würde, dass medizinische Fehler tatsächlich die dritthäufigste Todesursache in den USA sind (Makary und Daniel 2016). Darüber hinaus gaben die Autoren an, dass dies ihrer Meinung nach immer noch die tatsächliche Inzidenz von Todesfällen aufgrund von medizinischen Fehlern unterbewertet, „weil die zitierten Studien auf Fehlern beruhen, die aus 
dokumentierten Gesundheitsakten extrahiert werden konnten, und nur stationäre Todesfälle umfassen“ (Makary und Daniel 2016; meine Übersetzung S.M.). Die Forschung in weiteren Ländern hat deutlich gemacht, dass dies ein weltweites Problem ist (Wilson et al. 1995; Vincent et al. 2001; Sari et al. 2007; Schiøler et al. 2001; Davis et al. 2002; Baker et al. 2004; Michel et al. 2007; Aranaz-Andrés et al. 2008; Zegers et al. 2009; Soop et al. 2009). Die verfügbaren internationalen Daten deuten darauf hin, dass medizinische Fehler bei fast jedem zehnten Patienten zu Behinderungen oder zum Tod führen. Die wirtschaftlichen Kosten dieser Fehler sind dementsprechend erheblich, wobei längere Krankenhausaufenthalte, Einkommensverluste, Behinderungen und Rechtsstreitigkeiten in einigen Ländern jährlich viele Milliarden Dollar kosten (WHO 2009). Auch wenn die Patientensicherheit und die Qualitätsverbesserung in der Gesundheitsversorgung aufgrund dieser Statistiken zunehmend in den Mittelpunkt gerückt sind, gibt es nach wie vor erhebliche unberechtigte Unterschiede zwischen den Behandlungen, die routinemäßig in der Praxis durchgeführt werden, sowie Mängel bei allen Schlüsselaspekten einer qualitativ hochwertigen Gesundheitsversorgung: Sicherheit, Wirksamkeit, Effizienz, Gerechtigkeit, Rechtzeitigkeit und Patientenzentriertheit (Institute of Medicine 2013).

\section{3 "Learning Health Care" und Datenwissenschaft}

Angetrieben von diesen Bedenken und in einer Zeit, in der es durch informationstechnische Systeme im Gesundheitswesen zunehmend möglich wurde, große Datenmengen am Ort der Patientenversorgung zu erfassen, wurde 2007 im Institute for Medicine das Konzept des „Learning Health Care System“ (LHCS) vorgestellt (Institute of Medicine 2007). Der Bericht, der erste in einer Serie von mittlerweile 17 Berichten für die IOM Learning Health System Series, stellte einen neuen konzeptionellen Ansatz für die Integration von klinischer Forschung und klinischer Praxis vor, „bei dem die Wissensgenerierung so in den Kern der medizinischen Praxis eingebettet ist, dass sie ein natürlicher Auswuchs und ein Produkt des Prozesses der Gesundheitsversorgung ist und zu einer kontinuierlichen Verbesserung der Versorgung führt" (Institute of Medicine 2007).

Eine „Lernaktivität“ wurde definiert als: „,...] eine, die sowohl (1) die Erbringung von Gesundheitsdienstleistungen oder die Nutzung individueller Gesundheitsinformationen beinhaltet als auch (2) als angestrebtes Ziel hat zu lernen, wie die klinische Praxis oder der Wert, die Qualität oder die Effizienz der Systeme, Institutionen und Modalitäten, durch die Gesundheitsdienstleistungen erbracht werden, verbessert werden kann..." (Faden et al. 2013, S. 19; meine Übersetzung S.M.). Dies umfasst ein breites Spektrum von Aktivitäten (einschließlich pragmatischer klinischer Studien, vergleichender Wirksamkeitsforschung, Forschung und Praxis der Qualitätsverbesserung, Forschung und Praxis der Patientensicherheit, Qualitätssicherung usw.) (Faden et al. 2013).Ein wichtiger Aspekt des Lernens im Gesundheitswesen ist dabei die zunehmende Nutzung der großen Menge routinemäßig erhobener digitaler Gesundheitsdaten, die 
eine groß angelegte und mehrdimensionale Zusammenfassung und Analyse heterogener Datenquellen ermöglicht (Ienca et al. 2018). Die Zunahme dieser Daten hat auch bedeutende Möglichkeiten rund um die künstliche Intelligenz (KI) und Teilbereiche des maschinellen Lernens, der Verarbeitung natürlicher Sprache und der Robotik geschaffen. Es wird erwartet, dass diese Entwicklungen in der Datenwissenschaft das Gesundheitswesen verändern werden (Topol 2019). Mit der Fähigkeit, aus großen Sätzen klinischer Daten zu lernen, hat die Datenwissenschaft im Gesundheitswesen das Potenzial, ein breites Spektrum von Aktivitäten zu unterstützen, darunter Diagnostik (Liu et al. 2019), klinische Entscheidungsfindung (Shortliffe und Sepúlveda 2018), personalisierte Medizin (Schork 2019), klinische Forschung (Woo 2019), Arzneimittelentwicklung (Fleming 2018), Verwaltungsprozesse (Davenport und Kalakota 2019), und gesundheitliche Ungleichheiten (Chen et al. 2020). Leider ist die ,unbequeme Wahrheit”, dass die Möglichkeiten in der Wissenschaft der Gesundheitsdaten oft genau das bleiben Möglichkeiten (Panch et al. 2019). Organisationen des Gesundheitswesens verfügen oft nicht über die Dateninfrastruktur, die für die Erhebung der erforderlichen Daten benötigt wird, die Daten befinden sich oft in Silos entlang organisatorischer Grenzen, was die gemeinsame Nutzung dieser stark einschränkt, und unterschiedliche Datenschutz- und Einwilligungsanforderungen können die nationalen und internationalen Bemühungen um eine kooperative Gesundheitsforschung untergraben (Panch et al. 2019; McLennan et al. 2019). Diese Bedenken werden sich wahrscheinlich noch verstärken, wenn die verfügbaren Daten immer feinkörniger und vielfältiger werden (z. B. medizinische Bilder, physiologische Wellenformen usw.) (McLennan et al. 2019).

\subsection{Beispiel: Intensivmedizin und die MIMIC-Datenbank}

Intensivmedizin ist komplex, teuer und oft mit schlechten Ergebnissen verbunden (Gayat et al. 2018). Sie ist jedoch auch ein datenreiches Umfeld und stand bei den Bemühungen, die Datenwissenschaft zur Verbesserung der Gesundheitsversorgung zu nutzen, an vorderster Front. Im Zuge dessen wurden in einer Reihe von Ländern, kommerzielle und nichtkommerzielle ICU-Datenbanken entwickelt (Celi et al. 2013). Eine der bekanntesten Intensivpflege-Datenbanken, die zu Forschungszwecken genutzt wird, ist die Datenbank Medical Information Mart for Intensive Care (MIMIC), die vom Laboratory for Computational Physiology (LCP) am Massachusetts Institute of Technology gepflegt wird (Johnson et al. 2016). MIMIC-III ist die dritte Iteration der Datenbank, die klinische Daten im Zusammenhang mit der Aufnahme von 53.423 verschiedenen erwachsenen Patienten auf Intensivstationen des Beth Israel Deaconess Medical Center in Boston, Massachusetts, enthält. Die Daten umfassen Vitalparameter, Medikamentengabe, Labormessungen, Beobachtungen und Notizen des Pflegepersonals, Flüssigkeitsbilanz, Verfahrenscodes, Diagnosecodes, Bildgebungsberichte, Krankenhausaufenthaltsdauer, Überlebensdaten und mehr (Johnson et al. 2016). Die Datenbank wird Forschern weltweit für die Sekundäranalyse frei zugänglich gemacht, sobald 
einer Datennutzungsvereinbarung zugestimmt worden ist. MIMIC hat sich als eine äußerst wertvolle Ressource erwiesen, die die akademische und industrielle Forschung, Initiativen zur Qualitätsverbesserung und Hochschulkurse unterstützt. Auf das Erfordernis der Zustimmung des einzelnen Patienten hat das örtliche IRB seit über 10 Jahren verzichtet, weil das Projekt keine Auswirkungen auf die klinische Versorgung hat und weil die Daten vor ihrer Aufnahme in die Datenbank durch Entfernung aller geschützten Gesundheitsinformationen gemäß dem Health Insurance Portability and Accountability Act (HIPAA) de-identifiziert werden (Johnson et al. 2016). MIMIC ist jedoch eingeschränkt durch die Tatsache, dass es sich um eine monozentrische Datenbank handelt. Aus diesem Grund gab es in den letzten Jahren Versuche, das Projekt sowohl national als auch international auszuweiten (McLennan et al. 2019). Die Verknüpfung von Datenbanken über Zentren und Länder hinweg hat eine Reihe potenzieller Vorteile, u. a. die Möglichkeit, Modelle institutionenübergreifend zu validieren. Damit ließe sich einerseits feststellen, welche Ergebnisse institutionsspezifisch und welche verallgemeinerbar sind, andererseits die Erkenntnisgewinnung allgemein beschleunigen (Celi et al. 2013). Nationale Bemühungen haben zur Entwicklung der multizentrischen MIT-Philips eICU Collaborative Research Database (http://eicu-crd.mit.edu/) geführt. Diese enthält die Daten von Patienten, die auf Intensivstationen in mehr als 400 Krankenhäusern Vereinigten Staaten aufgenommen wurden. Obwohl auch mit internationalen Kooperationspartnern einige Fortschritte bei der Realisierung einer multizentrischen internationalen Datenbank für die Intensivpflege erzielt wurden, wurden die Bemühungen oftmals von lokalen Ethikkommissionen erschwert, die darüber beraten, ob die Zustimmung der Patienten zur Verwendung der Daten erforderlich ist (McLennan et al. 2019).

\section{Ethische Aufsicht über die Datenwissenschaft im Gesundheitswesen}

Wenn „Lernaktivitäten“ unter Nutzung der Gesundheitsdatenwissenschaft die kontinuierliche Verbesserung der Qualität der Gesundheitsversorgung verwirklichen und dadurch den Schaden für die Patienten zu verringern, die Gesundheit zu erhöhen, die Entscheidungsfindung der Patienten zu befähigen und die Gerechtigkeit $\mathrm{zu}$ verbessern können, würden sie die ethischen Kernprinzipien der Gesundheitsversorgung erfüllen (ABIM Foundation 2002; Beauchamp und Childress 2013). Neben der ethischen Begründung für solche Aktivitäten hat die normative und empirische Literatur jedoch auch verschiedene ethische Herausforderungen aufgezeigt. Da bei den Aktivitäten der Gesundheitsdatenwissenschaft routinemäßig erhobene Gesundheitsdaten verwendet werden, können Herausforderungen in Bezug auf die ethische Aufsicht aufgeworfen werden, darunter die Bestimmung, welche Aktivitäten einer ethischen Überprüfung durch eine Ethikkommission bedürfen (Morain und Kass 2016; Fiscella et al. 2015; Finkelstein et al. 2015; Kass und Pronovost 2011), und wann die Teilnehmer informiert und um ihre informierte Zustimmung gebeten werden sollten (Fiscella et al. 
2015; Finkelstein et al. 2015; Anderson et al. 2015; Pletcher et al. 2014; Mostert et al. 2016). Diese Ungewissheit birgt das Risiko, dass das System der ethischen Aufsicht die Bemühungen zur Verbesserung der Patientenversorgung untergräbt, indem es die Durchführung dieser Projekte übermäßig aufwändig macht (Tu et al. 2004; Miller und Emanuel 2008; Kass et al. 2008; Siegel und Alfano 2009; Taylor et al. 2010; Thompson et al. 2012).

\subsection{Forschung vs. Nicht-Forschung}

Nach verschiedenen Skandalen im zwanzigsten Jahrhundert wurden forschungsethische Aufsichtssysteme mit dem Ziel entwickelt, Patienten und andere Beteiligte vor Ausbeutung, Missbrauch oder ungerechtfertigten Risiken zu schützen (Kass et al. 2013). Infolgedessen wird von ethischen Aufsichtssystemen auf der ganzen Welt häufig eine scharfe Unterscheidung zwischen klinischer Forschung und klinischer Praxis getroffen. Forschung am Menschen erfordert in der Regel die Einholung der Genehmigung einer unabhängigen Ethikkommission sowie die vollständige Aufklärung der Teilnehmer und die Einholung ihrer schriftlichen Einwilligung. Für forschungsfremde Aktivitäten gibt es oft kein gleichwertiges Verfahren. Folglich kann die ethische Aufsicht über Aktivitäten, die dieselben Gesundheitsdaten verwenden, sehr unterschiedlich sein, wenn sie als „Forschung“ oder „Qualitätskontrolle“ klassifiziert werden.

Diese Aktivitäten können jedoch oft nicht zuverlässig voneinander abgegrenzt werden (McLennan et al. 2018). Während in den Vorschriften ,Forschung“ häufig als „,methodengesteuerte Suche nach verallgemeinerbarem Wissen” definiert wird, ist der Versuch, „Forschung“ und „Qualitätsverbesserung“ auf der Grundlage der methodischen Strenge (interne Validität) und der Verallgemeinerbarkeit (externe Validität) der Ergebnisse zu unterscheiden, in der Regel nicht überzeugend (Fiscella et al. 2015). Dies gilt häufig auch für die Aktivitäten „Forschung“ und „Qualitätskontrolle“ im Bereich der Gesundheitsdatenwissenschaft: Beide wenden rigorose wissenschaftliche Methoden an. Beide versuchen, Wissen aus der klinischen Praxis abzuleiten, um die Gesundheitsversorgung zu verbessern, und in vielen Fällen publizieren beide Aktivitäten ihre Ergebnisse öffentlich. Es ist daher schwierig, hierin eine Rechtfertigung dafür zu sehen, dass diese Aktivitäten einer sehr unterschiedlichen ethischen Aufsicht unterliegen (McLennan et al. 2018). Stattdessen sollte die ethische Aufsicht über die Aktivitäten besser von dem Risiko abhängen, das sie für die Teilnehmer darstellen, und nicht davon, ob die Aktivität als „Forschung“ bezeichnet wird (McLennan et al. 2018).

Es wird zunehmend anerkannt, dass für die meisten Initiativen, die auf die Verbesserung der Qualität der Gesundheitsversorgung abzielen, eine gewisse Form der ethischen Aufsicht wünschenswert ist (Perneger 2004). Es muss weiter darüber nachgedacht werden, wie datenwissenschaftliche Aktivitäten im Bereich der Gesundheitsversorgung stattfinden können. Sie sollen weder überreguliert (was die Bemühungen um eine Verbesserung der Patientenversorgung untergraben könnte), noch völlig 
ohne Aufsicht sein (was die Patienten möglicherweise ungerechtfertigten Risiken oder Belastungen aussetzt). Die meisten Strategien, die in der Literatur vorgeschlagen wurden, um die ethischen Fragen anzugehen, die sich aus dem Konflikt zwischen dem „Learning Health Care“ und den geltenden Vorschriften ergeben, zielen jedoch lediglich darauf ab, das derzeitige System zu verbessern.

Die wichtigsten Strategien lassen sich in drei große Gruppen einteilen: (1) Richtlinien und Verfahren: Klare und systematische interne Richtlinien und Verfahren, um zu bestimmen, welche Aktivitäten eine ethische Überprüfung erfordern (Morain und Kass 2016; Finkelstein et al. 2015), wie die gemeinsame Nutzung von Daten und der Datenschutz gehandhabt werden sollten (Docherty und Lone 2015), und wie Patienten routinemäßig und systematisch über durchgeführte Aktivitäten informiert werden können (Faden et al. 2013). (2) Schulung und Anleitung: Schulung und Anleitung für Mitglieder von Ethikkommissionen, damit sie lernen, wie ethische Prinzipien im Zusammenhang mit „Learning Health Care“ anzuwenden sind (Fiscella et al. 2015; Finkelstein et al. 2015; Kass und Pronovost 2011; Anderson et al. 2015; Thompson et al. 2012; Psek et al. 2015), und für Forscher, damit sie sich mit Ethikrichtlinien vertraut machen können (Ezzat et al. 2010). (3) Rationalisierung von Prozessen: Vereinfachung der ethischen Prüfung und des Zustimmungsverfahrens, um die Durchführung von Aktivitäten zu erleichtern, einschließlich der Einführung eines speziellen ethischen Prüfungsverfahrens (Anderson et al. 2015), der Standardisierung und Harmonisierung des ethischen Prüfungsverfahrens über mehrere Forschungsstandorte hinweg (Fiscella et al. 2015; Kass und Pronovost 2011; Anderson et al. 2015; Thompson et al. 2012; Ezzat et al. 2010), und der Straffung des Zustimmungsverfahrens (Fiscella et al. 2015; Anderson et al. 2015; Faden et al. 2014).

Das traditionelle Modell für die ethische Aufsicht, bei dem zeitlich begrenzte „Forschungs“--Projekte vor ihrer Durchführung einer prospektiven Bewertung unterzogen werden, ist für die kontinuierliche, integrierte und dynamische Natur der Gesundheitsdatenwissenschaften wenig geeignet. Anstatt das traditionelle Regelwerk zu verfeinern, scheint ein neues Modell der ethischen Aufsicht erforderlich zu sein. Das Konzept der „systemischen Aufsicht“ wurde unlängst vorgeschlagen, um den ethischen Herausforderungen in der großen Datengesundheitsforschung $\mathrm{zu}$ begegnen (Vayena und Blasimme 2018). Vayena und Blasimme schlagen vor, dass die Hauptmerkmale einer solchen „systemischen Aufsicht“ sind: „(1) die Fähigkeit, mit der Ungewissheit, die mit der Datenerhebung und Datennutzung einhergeht, durch adaptive und flexible Mechanismen zu umgehen; (2) die Fähigkeit, der erweiterten Zeitlichkeit datenbezogener Aktivitäten (von der Speicherung bis zur Re-analyse) durch dynamische Überwachung und Reaktionsfähigkeit zu begegnen; und schließlich (3) die Fähigkeit, mit der relationalen Natur großer biomedizinischer Daten durch Reflexivität und Inklusivität umzugehen“ (Vayena und Blasimme 2018, S. 8; meine Übersetzung S.M.). 


\subsection{Patienteneinwilligung und Forschungsausnahmen}

Patienten haben ein legitimes Interesse daran, den Zugang zu ihren Gesundheitsdaten und deren Verwendung zu kontrollieren. Ihre informierte Zustimmung wird oft für die Verwendung ihrer Daten für andere Zwecke als die, für die sie erhoben wurden, erforderlich sein. Das Erfordernis der individuellen informierten Einwilligung für die Verwendung pseudonymisierter (de-identifizierter) Daten in Datenbanken und Registern des Gesundheitswesens kann jedoch zu großen Kostensteigerungen führen und erhebliche Selektionsverzerrungen hervorrufen, die die Repräsentativität der Daten untergraben (Tu et al. 2004). Nichtsdestotrotz erlauben viele Länder einer Ethikkommission, auf das Erfordernis der Einwilligung für eine solche sekundäre Verwendung von Gesundheitsdaten $\mathrm{zu}$ verzichten, wenn bestimmte Bedingungen erfüllt sind. Diese Entscheidungen können jedoch oft nicht nur innerhalb der Länder, sondern auch zwischen den Ländern variieren. In einer Zeit zunehmender globaler kooperativer Bemühungen in der Gesundheitsforschung sind solche Unterschiede bei den Anforderungen an die Einwilligung zur Datenverarbeitung problematisch (McLennan et al. 2019).

Das Risiko einer stark lokalisierten ethischen Überprüfung an einem einzigen Standort, die die globale biomedizinische Datenforschung untergräbt, hat einige dazu veranlasst, die Harmonisierung der ethischen Überprüfung bestimmter Arten von datengetriebener Forschung durch die Schaffung multinationaler Verwaltungsstrukturen vorzuschlagen mit der Argumentation, dass ,,in einer Welt der großen Forschung und der großen Daten eine große Ethik erforderlich ist" (Dove et al. 2014). Dagegen ließe sich argumentieren, dass trotz der Notwendigkeit einer stärkeren globalen Harmonisierung lokale Ethikausschüsse weiterhin am besten in der Lage sind, die Werte und Normen lokaler Gemeinschaften zu beurteilen (McLennan et al. 2019). Es scheint jedoch, dass viele Ethikkommissionen derzeit unsicher sind, wie die Datenforschung verantwortungsbewusst reguliert werden sollte. Dies führt $\mathrm{zu}$ wachsenden Bedenken, dass Ethikkommissionen sich in einer Art „Überkompensierung“ zu sehr mit Risiken befassen und den Wert solcher Forschung für die Gesundheit der Bevölkerung nicht ausreichend berücksichtigen (Spector und Prainsack 2018). Ein Schlüsselfaktor, der solche risikoaversen Entscheidungen vorantreibt, scheint die Besorgnis hinsichtlich der Regulierung des Datenschutzes zu sein.

\subsubsection{Die Datenschutz-Grundverordnung (DSGVO)}

Im europäischen Kontext ist die Allgemeine Datenschutz-Grundverordnung (DSGVO) das wichtigste Rechtsinstrument und in allen EU-Mitgliedsstaaten direkt durchsetzbar. Die DSGVO gilt seit Mai 2016, trat aber erst ab dem 25. Mai 2018 in Kraft. Während frühe Entwürfe der DSGVO Bedenken auslösten, dass die Verordnung die Datenforschung stark einschränken könnte (Nyrén et al. 2014), wurde im endgültigen Text ein forschungsfreundlicherer Ansatz gewählt. Inzwischen wird davon ausgegangen, dass die DSGVO insgesamt wenig Einfluss auf die Datenforschung haben wird (Rumbold und Pierscionek 2017). Es bestehen jedoch weiterhin Bedenken, dass die DSGVO 
viele Organisationen in Bezug auf die gemeinsame Nutzung von Daten sehr risikoavers gemacht haben könnte, selbst wenn die Verordnung eine solche gemeinsame Nutzung erlaubt. Die DSGVO hat einige wichtige Änderungen eingeführt, die von der Gesundheitsdatenwissenschaft und den globalen Gesundheitsdatenbanken berücksichtigt werden müssen (siehe Tab. 1 für eine Zusammenfassung der wichtigsten Auswirkungen).

Tab. 1 Die wichtigsten Auswirkungen der DSGVO auf globale Gesundheitsdatenbanken

\begin{tabular}{l|l}
\hline Zusammenfassung der DSGVO-Vorschrift & $\begin{array}{l}\text { Auswirkungen auf die Datenwissenschaft im } \\
\text { Gesundheitswesen }\end{array}$ \\
\hline $\begin{array}{l}\text { Geltungsbereich: Die DSGVO gilt für alle } \\
\text { personenbezogenen Daten, die eine identi- } \\
\text { fizierte oder identifizierbare natürliche }\end{array}$ & $\begin{array}{l}\text { Da die DSGVO nicht zwischen anonymisierten } \\
\text { und anonymen Daten unterscheidet, werden } \\
\text { Aerson betreffen, jedoch nicht für anonyme } \\
\text { Informationen }\end{array}$ \\
$\begin{array}{l}\text { sorgung, bei denen identifizierbare Daten } \\
\text { zu Forschungszwecken gesammelt werden, } \\
\text { vom Anwendungsbereich der DSGVO } \\
\text { ausgeschlossen, wenn die Daten später } \\
\text { anonymisiert werden (Shabani und Borry 2018) }\end{array}$ \\
\hline
\end{tabular}

Pseudonymisierung: Pseudonymisierte Daten werden jetzt als personenbezogene Daten anerkannt, wenn sie durch die Verwendung zusätzlicher Informationen einer natürlichen Person zugeordnet werden können
Da pseudonymisierte Gesundheitsdaten in wissenschaftlichen Zusammenhängen sehr häufig verwendet werden, kann die Anerkennung pseudonymisierter Daten als personenbezogene Daten zu mehr Bürokratie führen, insbesondere für jene Länder, die bisher der Ansicht waren, dass pseudonymisierte Daten nicht als personenbezogene Daten fallen (Shabani und Borry 2018)

Besondere Kategorien personenbezogener Daten: Die Verarbeitung besonderer Kategorien personenbezogener Daten ("sensible personenbezogene Daten"), einschließlich genetischer Daten, biometrischer Daten und Gesundheitsdaten, ist verboten, es sei denn unter bestimmten Bedingungen

Die Wissenschaft im Bereich der Gesundheitsdaten, die pseudonymisierte sensible personenbezogene Daten verwendet, muss entweder die ausdrückliche Zustimmung der betroffenen Person einholen, oder die Daten müssen im Rahmen der in der DSGVO festgelegten Ausnahmeregelung für wissenschaftliche Forschung verarbeitet werden. Dies könnte u.U. ohne Zustimmung geschehen, wenn entsprechende technische und organisatorische Sicherheitsvorkehrungen getroffen werden (Shabani und Borry 2018)

\section{Räumlicher Anwendungsbereich: Die} DSGVO gilt für alle für die Datenverarbeitung Verantwortlichen und Auftragsverarbeiter, die personenbezogene Daten von in der EU ansässigen betroffenen Personen verarbeiten, unabhängig davon, ob die Verarbeitung in der EU stattfindet oder nicht.

\section{Globale Gesundheitsdatenbanken, die} außerhalb der EU angesiedelt sind, müssen der DSGVO entsprechen, wenn Gesundheitsdaten von Patienten mit Wohnsitz in der EU in die Datenbank aufgenommen werden 
Wichtig ist, dass Artikel 9(2)(j) der DSGVO eine Ausnahme für die wissenschaftliche Forschung für die Verarbeitung sensibler personenbezogener Daten vorsieht, die ohne Einwilligung erfolgen kann, wenn sie angemessenen Garantien unterliegt, zu denen auch die Pseudonymisierung gehören kann (siehe Artikel 89(1)). Obwohl diese Bestimmung existiert, scheinen Forscher und Forschungseinrichtungen in Europa jedoch zögerlich zu sein, sie zu nutzen. Ein möglicher Grund könnte sein, dass sie die Befürchtung haben, ihre nationalen Stellen könnten es ihnen schwer machen. In der Tat haben Konsortien, die im Rahmen des aktuellen H2020-Finanzierungsprogramms von der Europäischen Kommission finanziert werden, überwiegend andere, aufwändigere rechtliche Rechtfertigungen, wie z. B. eine informierte Zustimmung verwendet, anstelle die die Forschungsausnahme geltend zu machen (McLennan et al. 2020).

Darüber hinaus gibt es Bedenken, dass die DSGVO zu viel Spielraum für die Auslegung der Verordnung durch die Mitgliedstaaten in Bezug auf Schlüsselaspekte des Datenschutzes lässt. Das gilt auch für den Bereich der Pseudonymisierung, in dem den Mitgliedsländern die Entscheidung darüber, wann Daten als vollständig nicht identifizierbar gelten, welche weiteren Beschränkungen für die Verarbeitung sensibler Daten zu Forschungszwecken festgelegt werden sollten, welche Garantien und Bedingungen für die Verarbeitung von Daten im Rahmen der Forschungsfreistellung ausreichend sind etc. (Shabani und Borry 2018). Dies mag zwar zur Stärkung lokaler Werte und Normen beitragen, birgt jedoch die Gefahr, dass das Ziel der DSGVO, die gegenwärtige Heterogenität des Datenschutzes innerhalb der EU zu berücksichtigen, untergraben wird. Es wurde vorgeschlagen, dass die Aushandlung sektorspezifischer Verhaltenskodizes durch Berufsverbände dazu beitragen könnte, die Datenharmonisierung und -integration zu erleichtern (BBMRI-ERIC 2015). Ein solcher Verhaltenskodex könnte auch dazu beitragen, Datenbankbetreibern, Forschern und Ethikkommissionen Orientierungshilfen für notwendige organisatorische und technische Schutzvorkehrungen zum Schutz der Patientenrechte $\mathrm{zu}$ geben, ohne wichtige Forschung im Bereich der Gesundheitsdaten übermäßig zu behindern. Da die Gesundheitssysteme zunehmend auf klinische Routinedaten zurückgreifen, werden Fragen der ethischen Aufsicht über die Wissenschaft der Gesundheitsdaten wahrscheinlich nur noch stärker in den Vordergrund rücken und müssen proaktiv angegangen werden (McLennan et al. 2019).

\subsubsection{Datenwissenschaft im Gesundheitswesen und die Herausforderung der COVID-19-Pandemie}

Die COVID-19-Pandemie ist ein globales Gesundheitsproblem und erfordert eine länderübergreifende Zusammenarbeit in der Gesundheitsforschung. Eine wertvolle Informationsquelle für Forscher ist die große Menge an digitalen Gesundheitsdaten, die von den elektronischen Gesundheitsdatensystemen der Gesundheitsorganisationen kontinuierlich gesammelt werden. Solche digitalen Gesundheitsdaten liegen jedoch in der Regel in getrennten Systemen vor, weshalb u.U. die Forschung in vielen Ländern sind derzeit durch den Mangel an integrierten und umfassenden, öffentlich verfügbaren Daten auf Patientenebene zu COVID-19 stark beeinträchtigt ist. Forscher müssen 
Antworten aus begrenzten Analysen kleiner Fallserien ableiten, während große Mengen relevanter digitaler Gesundheitsdaten ungeprüft auf Krankenhaus-Servern auf der ganzen Welt liegen. Diese Situation hat zu Forderungen nach der Schaffung einer gemeinsamen, multinationalen COVID-19-Datenbank geführt, wobei auf die MIMIC-Datenbank als Modell für den öffentlichen Austausch von de-identifizierten elektronischen Gesundheitsdaten verwiesen wird (Cosgriff et al. 2020).

Während die Einrichtung von COVID-19-bezogenen Datenbanken aus der Forschungsperspektive Sinn macht, ist dies auch gerechtigkeitstheoretisch und gesellschaftlich angezeigt. Viele laufende Maßnahmen zur Eindämmung der Ausbreitung stellen pro-soziale Verhaltensweisen zur Hilfe und/oder zum Schutz anderer oder kollektiver Ressourcen wie der Gesundheitssysteme dar, die auf einer bestimmten Gemeinwohlvorstellung beruhen (McLennan et al. 2020). Auch Gesundheitsdatenbanken und Biobanken wurden früher als solidarische Bemühungen bezeichnet, und es wurden solidarische Steuerungsmodelle vorgeschlagen, um die pro-soziale Motivation vieler Menschen gegenüber solchen Ressourcen einzufangen, die gleichzeitig einen Teil der Last der üblichen restriktiven, autonomiebasierten Steuerungsmodelle vermeiden (Prainsack und Buyx 2013).

Da die Gesamtzahl der Todesfälle durch COVID-19 weltweit weiter zunimmt, ist die ethische und soziale Notwendigkeit einer raschen Eindämmung der Pandemie klar. Dies steht jedoch nicht im Widerspruch zu der Erfordernis, dass bei der Verwendung digitaler Gesundheitsdaten die Datenschutzbestimmungen sowie die Privatsphäre der und Vertraulichkeit gegenüber den Patienten gewahrt bleiben müssen (Ienca und Vayena 2020). Die DSGVO ist der wichtigste Rechtsrahmen für die gemeinsame Nutzung europäischer digitaler Gesundheitsdaten zu Forschungszwecken (McLennan et al. 2019). Gesundheitsorganisationen, die sich auf die individuelle Risikominimierung konzentrieren, drohen jedoch die Forschungsanstrengungen von COVID-19 zu untergraben.

Das European Data Protection Board hat die Bedeutung des Schutzes persönlicher Daten während der COVID-19-Pandemie betont. Es hat allerdings auch festgestellt: "Datenschutzbestimmungen (wie die DSGVO) behindern nicht die Maßnahmen, die im Kampf gegen die Coronavirus-Pandemie ergriffen werden“ (European Data Protection Board 2020). COVID-19 ist jedoch ein echter Test für die DSGVO. Es lässt sich argumentieren, dass es eine ethische Verpflichtung gibt, die Forschungsausnahmeklausel während der COVID-19-Pandemie zu nutzen, um globale kooperative Forschungsbemühungen zu unterstützen (McLennan et al. 2020). Dazu gehört auch, dass die Länder Gesundheitsorganisationen und Ermittler dabei unterstützen, sich im Kontext einer globalen Pandemie vertrauensvoll auf die Forschungsausnahme zu berufen. Neuere Forschungen in einigen europäischen Ländern deuten darauf hin, dass viele Menschen die sekundäre Verwendung ihrer Daten für die gesundheitsbezogene Forschung im Rahmen der Forschungsfreistellung akzeptieren würden, die auf pro-sozialen Beweggründen wie Solidarität beruht (Richter et al. 2019). Solidarität ist ein europäischer Wert, und hier bietet sich die Chance, diesen Wert zu verdeutlichen, indem der DSGVORechtsrahmen so genutzt wird, dass die Solidarität während der COVID-19-Pandemie nicht behindert, sondern sogar gefördert wird (McLennan 2020). 


\section{Literatur}

ABIM Foundation, American Board of Internal Medicine, ACP-ASIM Foundation, American College of Physicians-American Society of Internal Medicine \& European Federation of Internal Medicine (2002) Medical professionalism in the new millennium: a physician charter. Ann Intern Med 136(3):243-246

Anderson ML, Califf RM, Sugarman J (2015) Ethical and regulatory issues of pragmatic cluster randomized trials in contemporary health systems. Clin Trials 12:276-286

Aranaz-Andrés JM, Aibar-Remón C, Vitaller-Murillo J, Ruiz-López P, Limón-Ramírez R, TerolGarcía E, ENEAS work group (2008) Incidence of adverse events related to health care in Spain: results of the Spanish National Study of Adverse Events. J Epidemiol Commun Health 62:1022-1029

Baker GR, Norton PG, Flintoft V, Blais R, Brown A, Cox J, Etchells E, Ghali WA, Hébert P, Majumdar SR, O'Beirne M, Palacios-Derflingher L, Reid RJ, Sheps S, Tamblyn R (2004) The Canadian Adverse Events Study: the incidence of adverse events among hospital patients in Canada. Can Med Assoc J 170:1678-1686

Banja J (2005) Medical errors and medical narcissism. Jones and Bartlett Publishers, Boston

BBMRI-ERIC (2015) Position Paper on General Data Protection Regulation 2015. http://www. bbmri-eric.eu/wpcontent/uploads/BBMRI-ERIC-Position-Paper-General-Data-ProtectionRegulation-October-2015_rev1_title.pdf

Beauchamp TL, Childress JF (2013) Principles of biomedical ethics, 7. Aufl. Oxford University Press, Oxford

Chen IY, Joshi S, Ghassemi M (2020) Treating health disparities with artificial intelligence. Nat Med 26:16-17

Cosgriff CV, Ebner DK, Celi LA (2020) Data Sharing in the Era of COVID-19. Lancet Digital Health 2(5):e224

Davenport T, Kalakota R (2019) The potential for artificial intelligence in healthcare. Future Healthc J 6(2):94-98

Davis P, Lay-Yee R, Briant R, Ali W, Scott A, Schug S (2002). Adverse events in New Zealand public hospitals I: occurrence and impact. New Zealand Med J, 115(1167) http://www.nzma. org.nz/journal/115-1167/271/

Dove ES, Bartha M, Knoppers BM, Zawati MH (2014) Towards an ethics safe harbor for global biomedical research. J Law Biosci 1(1):3-51

European Data Protection Board (2020) Statement by the EDPB Chair on the processing of personal data in the context of the COVID-19 outbreak. 16 March 2020. https://edpb.europa.eu/ news/news/2020/statement-edpb-chair-processing-personal-data-context-covid-19-outbreak_en

Ezzat H, Ross S, Dadelszen P, Morris T, Liston R, Magee LA, CPN Collaborative Group (2010) Ethics review as a component of institutional approval for a multicentre continuous quality improvement project: the investigator's perspective. BMC Health Serv Res 10:223

Faden RR, Beauchamp TL, Kass NE (2014) Informed consent, comparative effectiveness, and learning health care. N Engl J Med 370:766-768

Faden RR, Kass NE, Goodman SN, Pronovost P, Tunis S, Beauchamp TL (2013) An Ethics Framework for a Learning Health Care System: A Departure from Traditional Research Ethics and Clinical Ethics. Hastings Cent Rep Special Report 43:S16-S27

Finkelstein JA, Brickman AL, Capron A, Ford DE, Gombosev A, Greene SM, Iafrate RP, Kolaczkowski L, Pallin SC, Pletcher MJ, Staman KL, Vazquez MA, Sugarman J (2015) Oversight on the borderline: quality improvement and pragmatic research. Clin Trials 12:457466 
Fiscella K, Tobin JN, Carroll JK, He H, Ogedegbe G (2015) Ethical oversight in quality improvement and quality improvement research: new approaches to promote a learning health care system. BMC Med Ethics 16:63

Fleming N (2018) How artificial intelligence is changing drug discovery. Nature 557:S55-S57

Hemkens LG, Contopoulos-Ioannidis DG, Ioannidis JP (2016) Routinely collected data and comparative effectiveness evidence: promises and limitations. CMAJ 188:E158-E164

Ienca M, Vayena E (2020) On the responsible use of digital data to tackle the COVID-19 pandemic. Nat Med. https://doi.org/10.1038/s41591-020-0832-5

Ienca M, Ferretti A, Hurst S, Puhan M, Lovis C, Vayena E (2018) Considerations for ethics review of big data health research: a scoping review. PLoS ONE 13(10):e0204937

Institute of Medicine (2013) Best care at lower cost: the path to continuously learning health care in America. The National Academies Press, Washington, DC

Institute of Medicine (2007) The learning healthcare system: workshop summary. The National Academies Press, Washington, DC

Institute of Medicine (2001) Crossing the Quality Chasm. Crossing the Quality Chasm: A New Health System for the 21st Century. National Academy Press, Washington, D.C.

Institute of Medicine (2000) To Err is human: building a safer health system. National Academy Press, Washington, D.C

Kass NE, Faden RR, Goodman SN, Pronovost P, Tunis S, Beauchamp TL (2013) The researchtreatment distinction: a problematic approach for determining which activities should have ethical oversight. Hastings Cent Rep, Spec No, S4-S15

Kass NE, Pronovost PJ (2011) Quality, safety, and institutional review boards: navigating ethics and oversight in applied health systems research. Am J Med Qual 26:157-159

Kass N, Pronovost PJ, Sugarman J, Goeschel CA, Lubomski LH, Faden R (2008) Controversy and quality improvement: lingering questions about ethics, oversight, and patient safety research. Jt Comm J Qual Patient Saf 34(6):349-353

Liu X, Faes L, Kale AU, Wagner SK, Fu DJ, Bruynseels A, Mahendiran T, Moraes G, Shamdas M, Kern C, Ledsam JR, Schmid MK, Balaskas K, Topol EJ, Bachmann LM, Keane PA, Denniston AK (2019) A comparison of deep learning performance against health-care professionals in detecting diseases from medical imaging: a systematic review and meta-analysis. Lancet Digital Health 1(6):e271-e297

Makary MA, Daniel M (2016) Medical error-the third leading cause of death in the US. BMJ 353:i2139

McLennan S, Celi LA Buyx A (2020) COVID-19: Putting the GDPR to the test Forthcoming

McLennan S, Shaw D, Celi LA (2019) The challenge of local consent requirements for global critical care databases. Intensive Care Med 45:246-248

McLennan S, Maritz R, Shaw D, Elger B (2018) The inconsistent ethical oversight of health care quality data in Switzerland. Swiss Med Weekly 148:w14637

Michel P, Quenon JL, Djihoud A, Tricaud-Vialle S, de Sarasqueta AM (2007) French national survey of inpatient adverse events prospectively assessed with ward staff. Qual Safety Health Care 16:369-377

Miller FG, Emanuel EJ (2008) Quality-improvement research and informed consent. N Engl J Med 358(8):765-767

Morain SR, Kass NE (2016) Ethics issues arising in the transition to learning health care systems: results from interviews with leaders from 25 health systems. EGEMS 4:11212

Mostert M, Bredenoord AL, Biesaart MC, van Delden JJ (2016) Big Data in medical research and EU data protection law: challenges to the consent or anonymise approach. Eur J Hum Genet 24:956-960 
Nyrén O, Stenbeck M, Grönberg H (2014) The European Parliament proposal for the new EU General Data Protection Regulation may severely restrict European epidemiological research. Eur J Epidemiol 29:227-230

Perneger T (2004) Why we need ethical oversight of quality improvement projects. Int J Qual Health Care 16:343-344

Pletcher MJ, Lo B, Grady D (2014) Informed consent in randomized quality improvement trials critical barrier for learning health systems. JAMA Intern Med 174:668-670

Prainsack B, Buyx A (2013) A Solidarity-based approach to the governance of research biobanks. Med Law Rev 21(1):71-91

Psek WA, Stametz RA, Stametz RA, Bailey-Davis LD, Davis D, Darer J, Faucett WA, Henninger DL, Sellers DC, Gerrity G (2015) Operationalizing the learning health care system in an integrated delivery system. eGEMs (Generating Evidence \& Methods to improve patient outcomes) 3(1):6

Richter G, Borzikowsky C, Lieb W, Schreiber S, Krawczak M, Buyx A (2019) Patient views on research use of clinical data without consent: Legal, but also acceptable? Eur J Hum Genet 27:841-847

Rumbold JMM, Pierscionek B (2017) The effect of the general data protection regulation on medical research. J Med Internet Res 19(2):e47

Sackett DL (1997) Evidence-based medicine. Semin Perinatol 21:3-5

Sari ABA, Sheldon TA, Cracknell A, Turnbull A, Dobson Y, Grant C, Gray W, Richardson A (2007) Extent, nature and consequences of adverse events: results of a retrospective casenote review in a large NHS hospital. Qual Safety Health Care 16(6):434-439

Shabani M, Borry P (2018) Rules for processing genetic data for research purposes in view of the new EU General Data Protection Regulation. Eur J Hum Genet 26:149-156

Sharpe VA (2004) Introduction: Accountability and Justice in Patient Safety Reform. In: Sharpe VA (Hrsg) Accountability and patient safety and policy reform. Georgetown University Press, Washington D.C., S 1-26

Schiøler T, Lipczak H, Pedersen BL, Mogensen TS, Bech KB, Stockmarr A, Svenning AR, Frølich A, Danish Adverse Event Study (2001) Incidence of adverse events in hospitals. A retrospective study of medical records. [Article in Danish] Ugeskr Laeger 163:5370-5378

Schork NJ (2019) Artificial intelligence and personalized medicine. Cancer Treat Res 178:265-283

Shortliffe EH, Sepúlveda MJ (2018) Clinical Decision Support in the Era of Artificial Intelligence. JAMA 320(21):2199-2200

Siegel MD, Alfano SL (2009) The ethics of quality improvement research. Crit Care Med 37(2):791-792

Soop M, Fryksmark U, Köster M, Haglund B (2009) The incidence of adverse events in Swedish hospitals: a retrospective medical record review study. International Journal of Quality in Health Care 21:285-291

Spector T, Prainsack B (2018) Ethics for healthcare data is obsessed with risk - not public benefits. The Conversation 2018. https://theconversation.com/ethics-for-healthcare-data-is-obsessedwith-risk-not-public-benefits-89575

Taylor HA, Pronovost PJ, Faden RR, Kass NE, Sugarman J (2010) The ethical review of health care quality improvement initiatives: findings from the field. Issue Brief (Commonw Fund) 95:1-12

Thompson DA, Kass N, Holzmueller C, Marsteller JA, Martinez EA, Gurses AP, Kanchuger M, Schwann N, Gibson CS, Bauer L, Pronovost PJ (2012) Variation in local institutional review board evaluations of a multicenter patient safety study. J Healthc Qual 34(4):33-39

Topol E (2019) Deep medicine: how artificial intelligence can make healthcare human again. Basic Books. ISBN-13: 978-15416446325

Tu JV, Willison DJ, Silver FL, Fang J, Richards JA, Laupacis A, Kapral MK, Investigators in the Registry of the Canadian Stroke Network (2004) Impracticability of informed consent in the Registry of the Canadian Stroke Network. N Engl J Med 350(14):1414-1421 
Vayena E, Blasimme A (2018) Health research with big data: time for systemic oversight. J Law Med Ethics 46(1):119-129

Vincent CA, Neale G, Woloshynowych M (2001) Adverse events in British hospitals: preliminary restrospective record review. BMJ 322:517-518

Wilson RM, Runciman WB, Gibberd RW, Harrison BT, Newby L, Hamilton JD (1995) The quality in Australian Health Care Study. Med J Aust 163:458-471

Woo M (2019) An AI boost for clinical trials. Nature 573:S100-S102

World Health Organization (2009) WHO Patient Safety Research. World Health Organization

Zegers M, de Bruijne MC, Wagner C, Hoonhout LH, Waaijman R, Smits M, Hout FA, Zwaan L, Christiaans-Dingelhoff I, Timmermans DR, Groenewegen PP, Wal G (2009) Adverse events and potentially preventable deaths in Dutch hospitals: results of a retrospective patient record review study. Quality Safety Health Care 18:297-302

Docherty AB, Lone NI (2015) Exploiting big data for critical care research. Curr Opin Crit Care 21:467-72.

Panch T, Mattie H, Celi LA (2019) The "inconvenient truth" about AI in healthcare. NPJ Digit Med 2:77

Etienne Gayat, Alain Cariou, Nicolas Deye, Antoine Vieillard-Baron, Samir Jaber, Charles Damoisel, Qin Lu, Xavier Monnet, Isabelle Rennuit, Elie Azoulay, Marc Léone, Heikel Oueslati, Bertrand Guidet, Diane Friedman, Antoine Tesnière, Romain Sonneville, Philippe Montravers, Sébastien Pili-Floury, Jean-Yves Lefrant, Jacques Duranteau, Pierre-François Laterre, Nicolas Brechot, Karine Chevreul, Morgane Michel, Bernard Cholley, Matthieu Legrand, Jean-Marie Launay, Eric Vicaut, Mervyn Singer, Matthieu Resche-Rigon, Alexandre Mebazaa, (2018) Determinants of long-term outcome in ICU survivors: results from the FROG-ICU study. Critical Care 22 (1)

Celi LA, Mark RG, Stone DJ, Montgomery RA (2013) "Big data" in the intensive care unit. Closing the data loop. Am J Respir Crit Care Med 187:1157-60

Johnson AE, Pollard TJ, Shen L, Lehman LW, Feng M, Ghassemi M, Moody B, Szolovits P, Celi LA, Mark RG (2016) MIMIC-III, a freely accessible critical care database. Sci Data 3:160035

Open Access Dieses Kapitel wird unter der Creative Commons Namensnennung 4.0 International Lizenz (http://creativecommons.org/licenses/by/4.0/deed.de) veröffentlicht, welche die Nutzung, Vervielfältigung, Bearbeitung, Verbreitung und Wiedergabe in jeglichem Medium und Format erlaubt, sofern Sie den/die ursprünglichen Autor(en) und die Quelle ordnungsgemäß nennen, einen Link zur Creative Commons Lizenz beifügen und angeben, ob Änderungen vorgenommen wurden.

Die in diesem Kapitel enthaltenen Bilder und sonstiges Drittmaterial unterliegen ebenfalls der genannten Creative Commons Lizenz, sofern sich aus der Abbildungslegende nichts anderes ergibt. Sofern das betreffende Material nicht unter der genannten Creative Commons Lizenz steht und die betreffende Handlung nicht nach gesetzlichen Vorschriften erlaubt ist, ist für die oben aufgeführten Weiterverwendungen des Materials die Einwilligung des jeweiligen Rechteinhabers einzuholen.

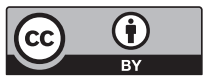

